\title{
Cogan's syndrome - A rare aortitis, difficult to diagnose but with therapeutic potential
}

\author{
Raiza Colodetti ${ }^{1}$, Guilherme Spina ${ }^{2}$, Tatiana Leal $^{3}$, Mucio Oliveira JR ${ }^{4}$, Alexandre Soeiro ${ }^{3 *}$ \\ ${ }^{1} \mathrm{MD}$ Cardiologist, Instituto do Coração (InCor), Hospital das Clínicas, Faculdade de Medicina da Universidade de São Paulo (HC-FMUSP), São Paulo, SP, Brazil \\ ${ }^{2}$ Assistant Physician at the Valvular Heart Disease Outpatient Clinic, InCor, HC-FMUSP, São Paulo, SP, Brazil \\ ${ }^{3}$ Assistant Physician at the Clinical Emergency Service, InCor, HC-FMUSP, São Paulo, SP, Brazil \\ ${ }^{4}$ Director of the Clinical Emergency Service, InCor, HC-FMUSP, São Paulo, SP, Brazil
}

Study conducted at Unidade Clínica de Emergência, Instituto do Coração (InCor), Hospital das Clínicas, Faculdade de Medicina da Universidade de São Paulo (HC-FMUSP), São Paulo, SP, Brazil

Article received: $8 / 18 / 2017$ Accepted for publication: 9/9/2017

*Correspondence: Address: Av. Dr. Enéas de

Carvalho Aguiar, 44

São Paulo, SP - Brazil

Postal code: 05403-900

alexandre.soeiro@bol.com.br

http://dx.doi.org/10.1590/1806-9282.63.12.1028

\section{SUMMARY}

The inflammation of aortic wall, named aortitis, is a rare condition that can be caused by a number of pathologies, mainly inflammatory or infectious in nature. In this context, the occurrence of combined audiovestibular and/or ocular manifestations eventually led to the diagnosis of Cogan's syndrome, making it the rare case, but susceptible to adequate immunosuppressive treatment and satisfactory disease control.

Keywords: chest pain, aortitis, Cogan's syndrome.

\section{INTRODUCTION}

Inflammation of the aortic wall, called aortitis, is an infrequent clinical condition that manifests itself with systemic symptoms and may cause precordial pain. ${ }^{1-4}$ One of the rheumatologic causes of aortitis is a rare disease called Cogan's syndrome. ${ }^{5}$ With approximately 300 cases reported in the world, it has no clearly defined etiology, pathophysiology, diagnosis or treatment, ${ }^{5}$ and affects from children to the elderly but mostly young adults (mean age 30 years). ${ }^{5}$ The occurrence of vestibular and ophthalmologic symptoms associated with the condition aided in the diagnosis of the case described, which, despite being rare, may respond to adequate immunosuppressive treatment achieving satisfactory disease control. ${ }^{6}$

\section{Case report}

This is the case of a 63 year-old male patient, Caucasian, born and living in the city of São Paulo, who attended the emergency department with a complaint of severe precordial chest pain described as tightness and irradiated to the mandible 2 hours before admission to hospital, combined with sweating and dyspnea. The patient reported the repeated occurrence of the symptoms, although less intense, for more than ten years, and that four years ago the episodes began to intensify. He was admitted to another service a week before for the same reason, where he underwent coronary angiography, showing no coronary obstruction, and an echocardiogram, which revealed a slight dilatation of the ascending aorta. In addition, the patient reported bilateral hypoacusis for 10 years (progressing to deafness in the right ear), rotational vertigo for 8 years and hyperemia, pain and eye tearing for two years. At admission, he did not regularly use any medications. He denied having risk factors for coronary heart disease or other comorbidities.

At physical examination, the patient presented a regular general condition, he was sweating and pale, with a heart rate of 75 beats per minute, breathing normally, with peripheral arterial oxygen saturation of $97 \%$, blood pressure of $130 \times 80 \mathrm{mmHg}$ in both arms, ictus not visible but palpable with fingertip in the normal position at the fourth intercostal space under the left midclavicular line, rhythmic heart sounds, no bruit heard and pulmonary auscultation with preserved vesicular murmur bilaterally without adventitious breath sounds. Pulse was heard bilaterally, wide and symmetrical. Jugular stasis was absent bilaterally at 45 degrees.

ECG results revealed sinus rhythm, without abnormalities suggestive of myocardial ischemia. At that moment, the 
diagnostic hypothesis of possible acute aortic dissection was raised. The initial treatment choice was to administer intravenous metoprolol and sodium nitroprusside, followed by immediate thoracic and abdominal aortic angiotomography.

Aortic angiotomography showed ectasia of ascending aorta (maximum caliper of $4.1 \mathrm{~cm}$ ), with no signs of dissection, and with slight parietal thickening of the ascending aorta, which remained with a small adjacent pericardial effusion, suggestive of aortitis (Figure 1).

From that moment, the inflammatory symptoms of probable aortitis began to be investigated. Transthoracic echocardiography showed grade 1 left ventricular diastolic dysfunction and discrete sinus and ascending aorta ectasia with preserved left ventricular systolic function. Laboratory tests revealed negative myocardial necrosis markers, leukocytosis $\left(14,070\right.$ leukocytes $/ \mathrm{mm}^{3}$ without left shift), increased C-reactive protein (CRP) $(245.97 \mathrm{mg} / \mathrm{L})$ and increased erythrocyte sedimentation rate (ESR) (49). Rheumatologic markers (rheumatoid factor, antinuclear factor, complement fractions $\mathrm{C} 3$ and $\mathrm{C} 4$, and anti-neutrophil cytoplasmic antibodies), serology for hepatitis B, hepatitis C and HIV, syphilis and cultures (blood and urine) were requested. All results were within normal range.

The patient underwent positron emission tomography (PET) to confirm the hypothesis of aortitis, which revealed a moderate diffuse uptake in the aortic wall from its root to the middle portion of the aortic arch, as well as a discrete/moderate diffuse image with thickening of the pericardial sac and effusion in its interior (Figure 2). Nuclear magnetic resonance imaging was also performed and showed only pericardial thickening.

Until that moment, the patient still had recurrence of the precordial pain episodes, being initially medicated with common analgesics and opioids. However, based on the adjuvant clinical history of ophthalmologic and audiovestibular involvement and the comple-

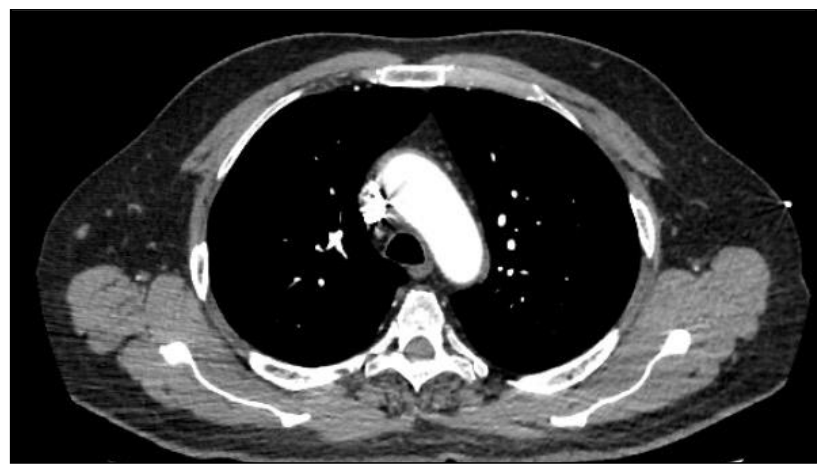

FIGURE 1 Angiotomography revealing parietal thickening in the ascending aorta. mentary exams performed, we raised the diagnostic hypothesis of Cogan's syndrome.

Treatment with prednisone $1 \mathrm{mg} / \mathrm{kg} /$ day was started on day 3 of hospitalization. From that day on, the patient progressed with important clinical improvement and did not present new episodes of precordialgia.

The patient was discharged on day 7 of hospitalization and day 5 of corticoid use; he was asymptomatic and his CRP level was $48.92 \mathrm{mg} / \mathrm{L}$. After two weeks, he returned for a follow-up visit still undergoing treatment with prednisone and remained asymptomatic, with CRP and ESR levels at $1.34 \mathrm{mg} / \mathrm{L}$ and 6 , respectively.

\section{Discussion}

Chest pain, as in our patient, is a common symptom in the emergency unit and deserves special attention due to the numerous diagnostic possibilities. Well-indicated anamnesis, physical examination and complementary exams are fundamental for the differential diagnosis. The diagnosis of acute coronary syndrome was removed since the results of the patient's coronary angiography did not reveal coronary obstructions and the ECG had no signs of myocardial ischemia. Changes in angiotomography, performed for the investigation of acute aortic dissection, raised the diagnostic hypothesis of aortitis.

Aortitis is an uncommon clinical condition, defined by inflammation of the aortic wall. ${ }^{1-4}$ It produces nonspecific symptoms such as fever, fatigue and weight loss, and may present with angina, aortic insufficiency and aortic dissection. ${ }^{2}$ It can be caused by infectious and non-infectious diseases, the latter being more common. ${ }^{1-3}$ Vasculitis, including giant cell arteritis and Takayasu's arteritis, are the most common causes of aortitis. Rarer conditions such as Cogan's syndrome are also mentioned in this context (Table 1). ${ }^{1,2}$

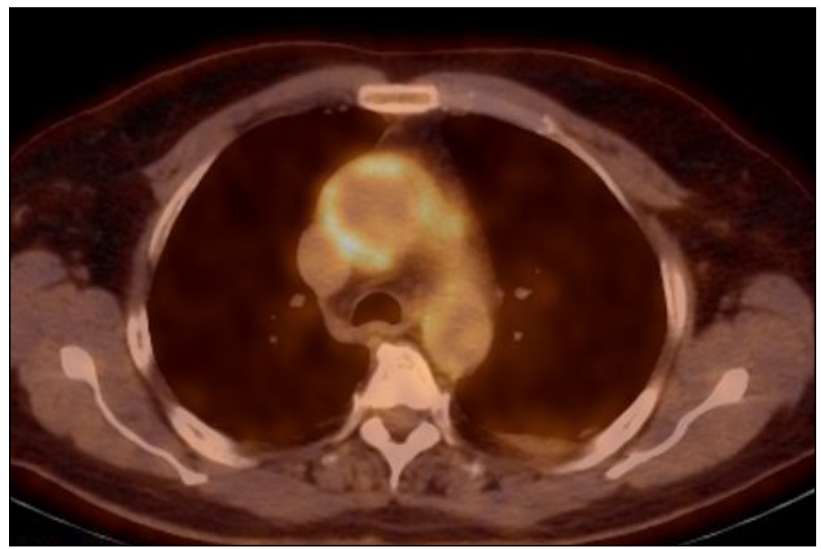

FIGURE 2 PET scan revealing moderate diffuse pattern capture in aortic walls. 
TABLE 1 Main causes of aortitis according to etiology.

\begin{tabular}{l} 
Non-infectious aortitis \\
\hline Large vessel vasculitis \\
\hline Giant cell arteritis \\
\hline Takayasu's arteritis \\
\hline Rheumatoid arthritis \\
\hline Systemic lupus erythematosus \\
\hline Spondyloarthropathies \\
\hline$\bullet \quad$ Ankylosing spondylitis \\
\hline$\bullet \quad$ Reiter's syndrome \\
\hline Other types of vasculitis \\
\hline ANCA-associated vasculitis \\
\hline$\bullet \quad$ Wegener's granulomatosis \\
\hline$\bullet \quad$ Polyarteritis nodosa \\
\hline$\bullet \quad$ Microscopic polyangiitis \\
\hline Behçet's disease \\
\hline Cogan's syndrome \\
\hline Relapsing polychondritis \\
\hline Sarcoidosis \\
\hline Idiopathic \\
\hline$\bullet \quad$ Idiopathic isolated aortitis \\
\hline$\bullet \quad$ Chronic periaortitis \\
\hline$\bullet \quad$ Inflamed aortic aneurysm \\
\hline Radiation-induced aortitis \\
\hline Infectious aortitis \\
\hline Bacteria \\
\hline$\bullet \quad$ Salmonella \\
\hline$\bullet \quad$ Staphylococcus spp \\
\hline$\bullet \quad$ Streptococcus spp \\
\hline Syphilis \\
\hline Mycobacteria \\
\hline Virus \\
\hline$\bullet$ HIV \\
\hline
\end{tabular}

The diagnosis of aortitis is difficult due to the lack of specificity of symptoms and low clinical suspicion, ${ }^{1}$ often obtained only with biopsy and histopathological analysis. ${ }^{4}$ Laboratory tests, such as inflammatory activity markers (C-reactive protein and ESR), aid in diagnosis when high levels are present, but are non-specific. ${ }^{2}$ Angiography has now been replaced by less invasive examinations, such as angiotomography and angioresonance. ${ }^{2}$ Both play a key role, since they detect thickening of the aortic wall, dilation and stenosis. Recently, PET tomography has been shown to be an important examination because it demonstrates increased aortic wall metabolism through the uptake of fluoride-18-labeled fluorordesoxyglucose. ${ }^{1,2,5}$ It has high sensitivity and specificity, reaching 92 and 100\%, respectively. ${ }^{1}$ It is useful not only to obtain a diagnosis, but also for follow-up, evaluating the response to treatment. ${ }^{1,5} \mathrm{In}$ our patient, the suspicion of aortitis arose due to the presence of parietal thickening in the ascending aorta at angiotomography. High CRP and ESR values corroborated our suspicion. The results of PET tomography, which demonstrated a moderate diffuse uptake of the aortic wall from its root to the middle portion of the aortic arch, were fundamental for the diagnosis of aortitis.

Defining the etiology of aortitis is not always an easy task, but it is essential for adequate treatment. It is based on epidemiological, clinical, laboratory and imaging data. General laboratory tests, such as blood count, electrolytes, renal function and liver function are part of the initial evaluation. ${ }^{2}$ It is imperative to rule out an infectious cause by collecting samples for culture and targeted research if there is any clinical suspicion of infection. ${ }^{2}$ The investigation of rheumatic diseases is also part of it, since they are the main causes of aortitis. Measuring rheumatologic markers such as rheumatoid factor, antinuclear factor, complement fractions (C3 and C4) and anti-neutrophil cytoplasmic antibodies is always indicated. ${ }^{2}$ In our case, the clinical history was fundamental for the diagnostic suspicion of Cogan's syndrome. In addition to aortitis, the patient had a history of bilateral hypoacusis in the last 10 years (progressing to deafness in the right ear), rotational vertigo for 8 years, and hyperemia, pain and eye tearing for 2 years. After ruling out any infectious causes of aortitis and other more frequent rheumatic diseases, the hypothesis of Cogan's syndrome became more evident.

Cogan's syndrome is an extremely rare condition, with approximately 300 cases reported worldwide..$^{5}$ It does not have well-defined etiology, diagnosis or treatment. It is believed that autoimmune mechanisms are involved, but its pathophysiology remains unknown. ${ }^{5}$ It affects from children to the elderly, mainly focusing on young adults (mean of 30 years). ${ }^{5}$ It is characterized by ocular changes such as hyperemia, pain, tearing, photophobia and foreign body sensation, accompanied by audiovestibular manifestations similar to Ménière's disease, characterized by vertigo, tinnitus, nystagmus, nausea and hearing loss with a sensorineural pattern, which can lead to deafness in a short term. ${ }^{6}$ The mean interval between ocular and auditory involvement is a few months, and sometimes years. ${ }^{6}$ Systemic manifestations are part of the syndrome in more than two thirds of the cases. Fever, fatigue, weight loss, vasculitis (including aortitis), pericarditis, arthralgia, lymphadenopathy, gastrointestinal and neurological symptoms may be present. ${ }^{5,6}$ 
Aortitis in Cogan's syndrome affects $10 \%$ of the individuals and has preference for the ascending aorta and aortic arch. ${ }^{4-6}$ It is characterized by mixed inflammatory infiltrate without granuloma, with areas of necrosis and destruction of the wall, ${ }^{4}$ and may cause dilation, aneurysm, aortic insufficiency and conduction blockages. ${ }^{5}$ The patient in our report is 63 years old, older than the mean of patients with Cogan's syndrome. Nevertheless, this does not rule out our diagnosis and in fact makes the case even more unusual. The symptoms presented by the patient, bilateral hypoacusis progressing to deafness in the right ear, rotational vertigo, and hyperemia, pain and eye tearing, are compatible with the ocular and audiovestibular manifestations observed in the syndrome. Symptom progression, in our case, occurred more slowly. The pattern of aortitis involvement in our patient, from the root of the aorta to the middle portion of the aortic arch, is more classically similar to the pattern of aortitis in most cases of Cogan's syndrome.

It is thus clear that the diagnosis of Cogan's syndrome is clinical, and there is no established diagnostic test. ${ }^{5}$ Due to the diagnostic difficulty, it is often recognized late, leading to a worse prognosis. ${ }^{5-6}$ It can progress slowly and gradually to chronicity or recurrences. ${ }^{5}$ Ocular changes are usually recurrent but present good resolution, usually causing no sequelae. ${ }^{5,6}$ Hearing changes, on the other hand, have a worse prognosis and progress to deafness in $50 \%$ of the cases. ${ }^{5,6}$ The presence of systemic manifestations such as vasculitis indicates a worse prognosis and a higher risk of complications, and may appear years after the initial symptoms. ${ }^{5,6}$ Our patient progressed in a manner compatible with that described in the literature. He eventually had deafness in his right ear but no ocular sequelae. Although he did not develop severe cardiovascular symptoms, recurrent precordial pain for 10 years and intensified in the past 4 years was probably due to recurrent and profuse aortitis.

Treatment of aortitis caused by Cogan's syndrome is not well established. There is no clinical trial comparing therapeutic options. ${ }^{5,6}$ Treatment consists basically of corticosteroids, and it may be necessary to associate with other immunosuppressants in more severe cases. ${ }^{5,6}$ In cases of complicated aortitis, surgical procedure may be necessary. ${ }^{5}$

In the case reported, the patient presented significant clinical improvement with the introduction of prednisone
$1 \mathrm{mg} / \mathrm{kg} /$ day, and no other immunosuppressants were required. He was discharged without symptoms and remained so until he returned for a medical visit two weeks later.

\section{Conclusion}

The case reports symptoms of precordial pain whose diagnosis was aortitis by Cogan's syndrome, a rare differential diagnosis. As mentioned above, Cogan's syndrome does not have a well-defined diagnosis or treatment, which can be recurrent and cause sequelae. The diagnosis of this patient was established years after the onset of the audiovestibular symptoms, when there were already auditory sequelae. Corticosteroid therapy yielded good results for the treatment of the vascular manifestation of the syndrome.

\section{Resumo}

Síndrome de Cogan - Uma aortite rara, de diagnóstico difícil, mas com potencial terapêutico

A inflamação da parede da aorta, denominada aortite, é uma condição clínica rara, que pode ser causada por diversas patologias, principalmente as de fundo inflamatório e/ou infeccioso. Nesse contexto, a ocorrência de sintomas vestibulares e oftalmológicos associados ao quadro remete ao diagnóstico de síndrome de Cogan, tornando o caso raro, mas passível de tratamento imunossupressor adequado e controle satisfatório da doença.

Palavras-chave: dor torácica, aortite, síndrome de Cogan.

\section{References}

1. Moyano JC, Magarolas MA, González EC, Cistaré XG, Castan EB. Patología aórtica no urgente: diagnóstico clínico-radiológico de la aortitis. Radiología. 2013; 55(6):469-82

2. Gornik HL, Creager MA. Aortic diseases: aortitis. Circulation. 2008; 117:3039-51.

3. Restrepo CS, Ocazionez D, Suri R, Vargas D. Aortitis imaging spectrum of the infectious and inflammatory conditions of the aorta. Radiographics. $2011 ; 31(2): 435-51$

4. Stone JR, Bruneval P, Angelini A, Bartoloni G, Basso C, Batoroeva L, et al. Consensus statement on surgical pathology of the aorta from the Society for Cardiovascular Pathology and the Association for European Cardiovascular Pathology: I. Inflammatory diseases. Cardiovasc Pathol. $2015 ; 24(5): 267-78$.

5. Montes S, Muguruza SR, Vina C, Olivé A. Síndrome de Cogan. Semin Fund Esp Reumatol. 2014; 15(1):19-24.

6. Murphy G, Sullivan MO, Harney S, Molloy M. Cogan's syndrome: present and future directions. Rheumato Int. 2009; 29(10):1117-21. 